Article

\title{
Applications of the Periodogram Method for Perturbed Block Toeplitz Matrices in Statistical Signal Processing
}

\author{
Jesús Gutiérrez-Gutiérrez * (D), Xabier Insausti ${ }^{\mathbb{D}}$ and Marta Zárraga-Rodríguez \\ Department of Biomedical Engineering and Sciences, Tecnun, University of Navarra, Paseo Manuel \\ Lardizábal 13, 20018 San Sebastián, Spain; xinsausti@tecnun.es (X.I.); mzarraga@tecnun.es (M.Z.-R.) \\ * Correspondence: jgutierrez@tecnun.es; Tel.: +34-943219877
}

Received: 15 February 2020; Accepted: 7 April 2020; Published: 14 April 2020

check for updates

\begin{abstract}
In this paper, we combine the periodogram method for perturbed block Toeplitz matrices with the Cholesky decomposition to give a parameter estimation method for any perturbed vector autoregressive (VAR) or vector moving average (VMA) process, when we only know a perturbed version of the sequence of correlation matrices of the process. In order to combine the periodogram method for perturbed block Toeplitz matrices with the Cholesky decomposition, we first need to generalize a known result on the Cholesky decomposition of Toeplitz matrices to perturbed block Toeplitz matrices.
\end{abstract}

Keywords: parameter estimation; periodogram method for perturbed block Toeplitz matrices; the Cholesky decomposition; vector autoregressive (VAR) processes; vector moving average (VMA) processes

\section{Introduction}

The Cholesky decomposition has been widely used in statistical signal processing. For instance, it has been used for parameter estimation of vector autoregressive (VAR) processes and for parameter estimation of vector moving average (VMA) processes. Specifically, the parameters of a VAR process can be directly obtained from the Cholesky decomposition of the inverses of its correlation matrices, and the parameters of a VMA process can be directly obtained from the Cholesky decomposition of its correlation matrices. However, when real-world problems are considered, what we usually know is a perturbed version of the sequence of correlation matrices of the process involved.

In this paper, we use the Cholesky decomposition to give a parameter estimation method for any perturbed VAR or VMA process, whenever the sequence of correlation matrices of the perturbed process is asymptotically equivalent to the sequence of correlation matrices of the original process in the Gray sense [1]. Specifically, our parameter estimation method combines the Cholesky decomposition with the periodogram method for perturbed block Toeplitz matrices presented in [2]. In order to combine them, we first need to generalize a result given in [3] on the Cholesky decomposition of Toeplitz matrices to perturbed block Toeplitz matrices.

The paper is organized as follows. In Section 2, we set up notation and we review the periodogram method for perturbed block Toeplitz matrices presented in [2]. In Section 3, we generalize a result given in [3] on the Cholesky decomposition of Toeplitz matrices to perturbed block Toeplitz matrices. In Section 4, we give a parameter estimation method for perturbed VAR and VMA processes. Our parameter estimation method for perturbed VMA processes is there also applied in another statistical signal processing problem, namely, in multiple-input multiple-output (MIMO) channel identification. 


\section{Preliminaries}

In this section, we set up notation and we review the periodogram method for perturbed block Toeplitz matrices presented in [2].

\subsection{Notation}

In this paper, $\mathbb{N}, \mathbb{Z}, \mathbb{R}$, and $\mathbb{C}$ denote the set of natural numbers (that is, the set of positive integers), the set of integer numbers, the set of real numbers, and the set of complex numbers, respectively. $\mathbb{C}^{M \times N}$ is the set of all $M \times N$ complex matrices, $I_{N}$ stands for the $N \times N$ identity matrix, $0_{M \times N}$ denotes the $M \times N$ zero matrix, and $V_{n}$ is the $n \times n$ Fourier unitary matrix, i.e.,

$$
\left[V_{n}\right]_{j, k}:=\frac{1}{\sqrt{n}} \mathrm{e}^{-\frac{2 \pi(j-1)(k-1)}{n} \mathrm{i}}, \quad j, k \in\{1, \ldots, n\},
$$

with $\mathrm{i}$ being the imaginary unit. We denote by $\lambda_{1}(A), \ldots, \lambda_{n}(A)$ the eigenvalues of an $n \times n$ Hermitian matrix $A$ arranged in decreasing order, $*$ denotes conjugate transpose, $\otimes$ is the Kronecker product, $E$ stands for expectation, and $\chi_{S}$ denotes the characteristic (or indicator) function of $S \subseteq \mathbb{R}$, that is,

$$
\chi_{S}(\omega):= \begin{cases}1 & \text { if } \omega \in S \\ 0 & \text { otherwise }\end{cases}
$$

If $x_{k} \in \mathbb{C}^{N \times 1}$ for all $k \in\{1, \ldots, n\}$, then $x_{n: 1}$ is the $n N$-dimensional vector given by

$$
x_{n: 1}=\left(\begin{array}{c}
x_{n} \\
x_{n-1} \\
\vdots \\
x_{1}
\end{array}\right) .
$$

If $x_{n}$ is a (complex) random $N$-dimensional vector for all $n \in \mathbb{N},\left\{x_{n}\right\}$ denotes the corresponding (complex) random $N$-dimensional vector process.

Let $A_{n}$ and $B_{n}$ be $n M \times n N$ matrices for all $n \in \mathbb{N}$. We write $\left\{A_{n}\right\} \sim\left\{B_{n}\right\}$ if the sequences $\left\{A_{n}\right\}$ and $\left\{B_{n}\right\}$ are asymptotically equivalent (i.e., $\left\{\left\|A_{n}\right\|_{2}\right\}$ and $\left\{\left\|B_{n}\right\|_{2}\right\}$ are bounded and $\lim _{n \rightarrow \infty} \frac{\left\|A_{n}-B_{n}\right\|_{F}}{\sqrt{n}}=0$ with $\|\cdot\|_{2}$ and $\|\cdot\|_{F}$ being the spectral norm and the Frobenius norm, respectively). We recall that the concept of asymptotically equivalent sequences of matrices was introduced by Gray in [1] for the case in which $M=N=1$.

If $F: \mathbb{R} \rightarrow \mathbb{C}^{M \times N}$ is a continuous $2 \pi$-periodic function, we denote by $T_{n}(F)$ the block Toeplitz matrix generated by $F$ whose blocks are given by

$$
\left[T_{n}(F)\right]_{j, k}:=\mathrm{F}_{j-k}, \quad n \in \mathbb{N}, \quad j, k \in\{1, \ldots, n\},
$$

where $\left\{\mathrm{F}_{k}\right\}_{k \in \mathbb{Z}}$ is the sequence of Fourier coefficients of $F$, that is,

$$
\mathrm{F}_{k}:=\frac{1}{2 \pi} \int_{0}^{2 \pi} \mathrm{e}^{-k \omega \mathrm{i}} F(\omega) d \omega \quad \forall k \in \mathbb{Z}
$$

\subsection{The Periodogram Method for Perturbed Block Toeplitz Matrices}

The following theorem, which was given in ([2], Theorem 4), provides a method to estimate the generating function $F$ when we only know a perturbed version of the sequence of block Toeplitz matrices $\left\{T_{n}(F)\right\}$, namely, we only know a sequence of matrices $\left\{A_{n}\right\}$ which is asymptotically equivalent to $\left\{T_{n}(F)\right\}$. 
Theorem 1. Let $A_{n}$ be an $n M \times n N$ matrix for all $n \in \mathbb{N}$. Suppose that there exists a continuous $2 \pi$-periodic function $F: \mathbb{R} \rightarrow \mathbb{C}^{M \times N}$ such that $\lim _{n \rightarrow \infty} \frac{\left\|A_{n}-T_{n}(F)\right\|_{F}}{\sqrt{n}}=0$. Then

$$
\lim _{n \rightarrow \infty} \frac{1}{2 \pi} \int_{0}^{2 \pi}\left\|\widehat{P}_{A_{n}}(\omega)-F(\omega)\right\|_{F}^{2} d \omega=0
$$

where $\widehat{P}_{A_{n}}: \mathbb{R} \rightarrow \mathbb{C}^{M \times N}$ is the $2 \pi$-periodic step function given by

$$
\widehat{P}_{A_{n}}(\omega):=\sum_{k=1}^{n} \chi_{\left[\frac{2 \pi(k-1)}{n}, \frac{2 \pi k}{n}\right)}(\omega)\left[\left(V_{n} \otimes I_{M}\right)^{*} A_{n}\left(V_{n} \otimes I_{N}\right)\right]_{k, k} \quad \forall \omega \in[0,2 \pi) .
$$

Moreover, if $F$ is a trigonometric polynomial there exists $K \in[0, \infty)$ such that

$$
\sqrt{\frac{1}{2 \pi} \int_{0}^{2 \pi}\left\|\widehat{P}_{A_{n}}(\omega)-F(\omega)\right\|_{F}^{2} d \omega} \leq \frac{\left\|A_{n}-T_{n}(F)\right\|_{F}}{\sqrt{n}}+\frac{K}{\sqrt{n}} \quad \forall n \in \mathbb{N} .
$$

The estimation method of the generating function $F$ provided in Theorem 1 consists of the sequence of functions $\left\{\widehat{P}_{A_{n}}\right\}$. Observe that from Equation (1) the squared error made, when $F$ is estimated (approximated) by $\widehat{P}_{A_{n}}$, tends to zero as $n$ grows.

The correlation matrix of a random vector is a positive semidefinite matrix. Furthermore, if $A$ is a positive semidefinite matrix, then there exists a zero-mean random vector whose correlation matrix is $A$. Therefore, $\left\{T_{n}(F)\right\}$ is a sequence of positive semidefinite matrices if and only if $\left\{T_{n}(F)\right\}$ is the sequence of correlation matrices of certain wide sense stationary (WSS) $N$-dimensional vector process (we recall that a random vector process $\left\{x_{n}\right\}$ is said to be WSS if its correlation matrices $E\left(x_{n: 1} x_{n: 1}^{*}\right)$ are block Toeplitz and its random vectors $x_{n}$ have the same mean). If $\left\{T_{n}(F)\right\}$ is the sequence of correlation matrices of a WSS vector process, the generating function $\mathrm{F}$ is called the power spectral density (PSD) of the process. Therefore, Theorem 1 provides a method to estimate the PSD (a spectral estimation method) of any WSS vector process, when we only know a perturbed version of its sequence of correlation matrices. This spectral estimation method is a modified version of the (averaged) periodogram method, because if $N=1$ then

$$
\begin{aligned}
& \widehat{P}_{T_{n}}\left(\frac{2 \pi(h-1)}{n}\right)=\left[V_{n}^{*} T_{n} V_{n}\right]_{h, h}=\sum_{k=1}^{n}\left[V_{n}^{*} T_{n}\right]_{h, k}\left[V_{n}\right]_{k, h}=\sum_{k=1}^{n}\left[V_{n}\right]_{k, h} \sum_{j=1}^{n}\left[V_{n}^{*}\right]_{h, j}\left[T_{n}\right]_{j, k} \\
& =\sum_{j, k=1}^{n}\left[V_{n}\right]_{k, h} \overline{\left[V_{n}\right]_{j, h}}\left[T_{n}\right]_{j, k}=\frac{1}{n} \sum_{j, k=1}^{n} \mathrm{e}^{\frac{2 \pi(j-k)(h-1)}{n}} \mathrm{i}\left[T_{n}\right]_{j, k}=P_{T_{n}}\left(\frac{2 \pi(h-1)}{n}\right), \quad n \in \mathbb{N}, \quad h \in\{1, \ldots, n\},
\end{aligned}
$$

where $\left\{P_{T_{n}}\right\}$ is the conventional spectral estimator, which is also known as the method of (averaged) periodogram or as the Bartlett method (see, e.g., [4]), defined as

$$
P_{T_{n}}(\omega):=\frac{1}{n} \sum_{j, k=1}^{n} \mathrm{e}^{(j-k) \omega \mathrm{i}}\left[T_{n}\right]_{j, k}, \quad n \in \mathbb{N}, \quad \omega \in \mathbb{R} .
$$

\section{A Note on the Cholesky Decomposition of Perturbed Block Toeplitz Matrices}

We recall that if $A$ is an $n \times n$ positive definite matrix, then there exists a unique $n \times n$ lower triangular matrix $L$ with $[L]_{j, j}>0$ for all $j \in\{1, \ldots, n\}$ satisfying that $A=L L^{*}$. This decomposition of $A\left(A=L L^{*}\right)$ is called the Cholesky decomposition of $A$. In ([3], Section 6.3) Gray gave a result on the Cholesky decomposition of Toeplitz matrices. The following theorem generalizes this result to perturbed block Toeplitz matrices. Furthermore, unlike in ([3], Section 6.3) we also give the convergence speed of our result.

Theorem 2. Consider a continuous $2 \pi$-periodic function $F: \mathbb{R} \rightarrow \mathbb{C}^{N \times N}$ whose sequence of Fourier coefficients $\left\{F_{k}\right\}_{k \in \mathbb{Z}}$ satisfies that $F_{0}$ is lower triangular with $\left[F_{0}\right]_{j, j}>0$ for all $j \in\{1, \ldots, N\}$ and $F_{-k}=0_{N \times N}$ for all $k \in \mathbb{N}$. Suppose that $A_{n}$ is an $n N \times n N$ positive definite matrix for all $n \in \mathbb{N}$ with $\left\{A_{n}\right\} \sim\left\{T_{n}(F)\left(T_{n}(F)\right)^{*}\right\}$ 
(or equivalently, $\left\{A_{n}\right\} \sim\left\{T_{n}\left(F F^{*}\right)\right\}$, where $\left.F F^{*}(\omega)=F(\omega)(F(\omega))^{*}, \omega \in \mathbb{R}\right)$. Let $A_{n}=L_{n} L_{n}^{*}$ be the Cholesky decomposition of $A_{n}$ for all $n \in \mathbb{N}$. If $\left\{L_{n}\right\}$ and $\left\{T_{n}(F)\right\}$ are stable (that is, $\left\{\left\|L_{n}^{-1}\right\|_{2}\right\}$ and $\left\{\left\|\left(T_{n}(F)\right)^{-1}\right\|_{2}\right\}$ are bounded) then

$$
\left\{L_{n}\right\} \sim\left\{T_{n}(F)\right\}
$$

Moreover, there exists $K \in[0, \infty)$ such that

$$
\frac{\left\|L_{n}-T_{n}(F)\right\|_{F}}{\sqrt{n}} \leq K \frac{\left\|A_{n}-T_{n}(F)\left(T_{n}(F)\right)^{*}\right\|_{F}}{\sqrt{n}} \quad \forall n \in \mathbb{N} .
$$

Proof. Applying ([5], Lemma 4.2) and ([5], Theorem 6.2) yields $\left\{T_{n}(F)\left(T_{n}(F)\right)^{*}\right\}=\left\{T_{n}(F) T_{n}\left(F^{*}\right)\right\} \sim$ $\left\{T_{n}\left(F F^{*}\right)\right\}$ (we recall that ([5], Theorem 6.2) was previously given for Hermitian generating functions (see, e.g., [6,7], or ([8], Theorem 2))). Hence, since the relation $\sim$ is symmetric and transitive (see ([5], Lemma 3.1)), $\left\{A_{n}\right\} \sim\left\{T_{n}(F)\left(T_{n}(F)\right)^{*}\right\}$ if and only if $\left\{A_{n}\right\} \sim\left\{T_{n}\left(F F^{*}\right)\right\}$.

The sequence $\left\{\left\|T_{n}(F)\right\|_{2}\right\}$ is bounded (see, e.g., ([5], Theorem 4.3) or ([9], Corollary 4.2)). As $\left\{\left\|A_{n}\right\|_{2}\right\}$ is bounded and

$$
\left\{\left\|L_{n}\right\|_{2}\right\}=\left\{\left\|L_{n}^{*}\right\|_{2}\right\}=\left\{\sqrt{\lambda_{1}\left(L_{n} L_{n}^{*}\right)}\right\}=\left\{\sqrt{\left\|L_{n} L_{n}^{*}\right\|_{2}}\right\}=\left\{\sqrt{\left\|A_{n}\right\|_{2}}\right\},
$$

$\left\{\left\|L_{n}\right\|_{2}\right\}$ is also bounded. Consequently, to finish the proof we only need to show Equation (3), or equivalently, we only need to show that there exists $K \in[0, \infty)$ such that

$$
\left\|L_{n}-T_{n}(F)\right\|_{F} \leq K\left\|A_{n}-T_{n}(F)\left(T_{n}(F)\right)^{*}\right\|_{F} \quad \forall n \in \mathbb{N} .
$$

We have

$$
\begin{aligned}
\left\|L_{n}-T_{n}(F)\right\|_{F} & =\left\|T_{n}(F)\left(T_{n}(F)\right)^{-1} L_{n}-T_{n}(F)\right\|_{F} \\
& \leq\left\|T_{n}(F)\right\|_{2}\left\|\left(T_{n}(F)\right)^{-1} L_{n}-I_{n N}\right\|_{F} \leq\left\|T_{n}(F)\right\|_{2}\left(\left\|\left(T_{n}(F)\right)^{-1} L_{n}-D_{n}\right\|_{F}+\left\|D_{n}-I_{n N}\right\|_{F}\right),
\end{aligned}
$$

where $D_{n}$ denotes the $n N \times n N$ diagonal matrix satisfying that $\left[D_{n}\right]_{j, j}=\left[\left(T_{n}(F)\right)^{-1} L_{n}\right]_{j, j}$ for all $j \in\{1, \ldots, n N\}$ and $n \in \mathbb{N}$. Since $T_{n}(F)$ is lower triangular for all $n \in \mathbb{N},\left(T_{n}(F)\right)^{-1}$ is lower triangular for all $n \in \mathbb{N}$ (see, e.g., ([10], p. 44)), and therefore,

$$
1=\left[I_{n N}\right]_{j, j}=\left[T_{n}(F)\left(T_{n}(F)\right)^{-1}\right]_{j, j}=\sum_{k=1}^{n N}\left[T_{n}(F)\right]_{j, k}\left[\left(T_{n}(F)\right)^{-1}\right]_{k, j}=\left[T_{n}(F)\right]_{j, j}\left[\left(T_{n}(F)\right)^{-1}\right]_{j, j}
$$

for all $j \in\{1, \ldots, n N\}$ and $n \in \mathbb{N}$. Thus,

$$
\left[D_{n}\right]_{j, j}=\left[\left(T_{n}(F)\right)^{-1} L_{n}\right]_{j, j}=\sum_{k=1}^{n N}\left[\left(T_{n}(F)\right)^{-1}\right]_{j, k}\left[L_{n}\right]_{k, j}=\left[\left(T_{n}(F)\right)^{-1}\right]_{j, j}\left[L_{n}\right]_{j, j}=\frac{\left[L_{n}\right]_{j, j}}{\left[T_{n}(F)\right]_{j, j}}>0
$$

for all $j \in\{1, \ldots, n N\}$ and $n \in \mathbb{N}$, and hence,

$$
\begin{aligned}
& \left\|D_{n}-I_{n N}\right\|_{F} \\
& =\sqrt{\sum_{j=1}^{n N}\left|\left[D_{n}-I_{n N}\right]_{j, j}\right|^{2}}=\sqrt{\sum_{j=1}^{n N}\left|\left[D_{n}\right]_{j, j}-1\right|^{2}}=\sqrt{\sum_{j=1}^{n N}\left|\frac{\left(\left[D_{n}\right]_{j, j}\right)^{2}-1}{\left[D_{n}\right]_{j, j}+1}\right|^{2}}=\sqrt{\sum_{j=1}^{n N} \frac{\left|\left[D_{n} D_{n}\right]_{, j}-1\right|^{2}}{\left(\left[D_{n}\right]_{j, j}+1\right)^{2}}} \\
& \leq \sqrt{\sum_{j=1}^{n N}\left|\left[D_{n} D_{n}\right]_{j, j}-1\right|^{2}}=\left\|D_{n} D_{n}-I_{n N}\right\|_{F}=\left\|D_{n} D_{n}^{*}-I_{n N}\right\|_{F}=\left\|D_{n} D_{n}^{*}-\left(T_{n}(F)\right)^{-1} L_{n}\left(L_{n}\right)^{-1} T_{n}(F)\right\|_{F} \\
& \leq\left\|D_{n} D_{n}^{*}-\left(T_{n}(F)\right)^{-1} L_{n} D_{n}^{*}\right\|_{F}+\left\|\left(T_{n}(F)\right)^{-1} L_{n} D_{n}^{*}-\left(T_{n}(F)\right)^{-1} L_{n}\left(L_{n}\right)^{-1} T_{n}(F)\right\|_{F} \\
& \leq\left\|D_{n}-\left(T_{n}(F)\right)^{-1} L_{n}\right\|_{F}\left\|D_{n}^{*}\right\|_{2}+\left\|\left(T_{n}(F)\right)^{-1} L_{n}\right\|_{2}\left\|D_{n}^{*}-\left(L_{n}\right)^{-1} T_{n}(F)\right\|_{F} \\
& =\left\|D_{n}-\left(T_{n}(F)\right)^{-1} L_{n}\right\|_{F}\left\|D_{n}\right\|_{2}+\left\|\left(T_{n}(F)\right)^{-1} L_{n}\right\|_{2}\left\|D_{n}-\left(T_{n}(F)\right)^{*}\left(L_{n}^{*}\right)^{-1}\right\|_{F} \\
& \leq\left\|D_{n}-\left(T_{n}(F)\right)^{-1} L_{n}\right\|_{F}\left\|D_{n}\right\|_{2} \\
& \\
& \quad+\left\|\left(T_{n}(F)\right)^{-1}\right\|_{2}\left\|L_{n}\right\|_{2}\left(\left\|D_{n}-\left(T_{n}(F)\right)^{-1} L_{n}\right\|_{F}+\left\|\left(T_{n}(F)\right)^{-1} L_{n}-\left(T_{n}(F)\right)^{*}\left(L_{n}^{*}\right)^{-1}\right\|_{F}\right) \quad \forall n \in \mathbb{N} .
\end{aligned}
$$


As $T_{n}(F)$ and $L_{n}$ are lower triangular for all $n \in \mathbb{N},\left(T_{n}(F)\right)^{-1} L_{n}$ and $L_{n}^{-1} T_{n}(F)$ are lower triangular for all $n \in \mathbb{N}$ (see, e.g., ([11], p. 240)). Consequently, $\left(T_{n}(F)\right)^{*}\left(L_{n}^{*}\right)^{-1}$ is upper triangular for all $n \in \mathbb{N}$, and therefore,

$$
\begin{aligned}
& \left\|\left(T_{n}(F)\right)^{-1} L_{n}-D_{n}\right\|_{F}=\sqrt{\left\|\left(T_{n}(F)\right)^{-1} L_{n}-D_{n}\right\|_{F}^{2}} \leq \sqrt{\left\|\left(T_{n}(F)\right)^{-1} L_{n}-D_{n}\right\|_{F}^{2}+\left\|D_{n}-\left(T_{n}(F)\right)^{*}\left(L_{n}^{*}\right)^{-1}\right\|_{F}^{2}} \\
& =\sqrt{\left\|\left(T_{n}(F)\right)^{-1} L_{n}-\left(T_{n}(F)\right)^{*}\left(L_{n}^{*}\right)^{-1}\right\|_{F}^{2}}=\left\|\left(T_{n}(F)\right)^{-1} L_{n}-\left(T_{n}(F)\right)^{*}\left(L_{n}^{*}\right)^{-1}\right\|_{F} \quad \forall n \in \mathbb{N} .
\end{aligned}
$$

Combining Equations (5), (6), and (7) yields

$$
\begin{aligned}
& \left\|L_{n}-T_{n}(F)\right\|_{F} \\
& \leq\left\|T_{n}(F)\right\|_{2}\left(1+\left\|D_{n}\right\|_{2}+2\left\|\left(T_{n}(F)\right)^{-1}\right\|_{2}\left\|L_{n}\right\|_{2}\right)\left\|\left(T_{n}(F)\right)^{-1} L_{n}-\left(T_{n}(F)\right)^{*}\left(L_{n}^{*}\right)^{-1}\right\|_{F} \\
& \leq\left\|T_{n}(F)\right\|_{2}\left(1+\left\|D_{n}\right\|_{2}+2\left\|\left(T_{n}(F)\right)^{-1}\right\|_{2}\left\|L_{n}\right\|_{2}\right)\left\|\left(T_{n}(F)\right)^{-1}\right\|_{2}\left\|L_{n}-T_{n}(F)\left(T_{n}(F)\right)^{*}\left(L_{n}^{*}\right)^{-1}\right\|_{F} \\
& \leq\left\|T_{n}(F)\right\|_{2}\left(1+\left\|D_{n}\right\|_{2}+2\left\|\left(T_{n}(F)\right)^{-1}\right\|_{2}\left\|L_{n}\right\|_{2}\right)\left\|\left(T_{n}(F)\right)^{-1}\right\|_{2}\left\|L_{n} L_{n}^{*}-T_{n}(F)\left(T_{n}(F)\right)^{*}\right\|_{F}\left\|\left(L_{n}^{*}\right)^{-1}\right\|_{2} \\
& =K_{n}\left\|A_{n}-T_{n}(F)\left(T_{n}(F)\right)^{*}\right\|_{F}
\end{aligned}
$$

with $K_{n}=\left\|T_{n}(F)\right\|_{2}\left(1+\left\|D_{n}\right\|_{2}+2\left\|\left(T_{n}(F)\right)^{-1}\right\|_{2}\left\|L_{n}\right\|_{2}\right)\left\|\left(T_{n}(F)\right)^{-1}\right\|_{2}\left\|L_{n}^{-1}\right\|_{2}$ for all $n \in \mathbb{N}$. To prove Equation (4) we only need to show that $\left\{K_{n}\right\}$ is bounded, or equivalently, we only need to show that $\left\{\left\|D_{n}\right\|_{2}\right\}$ is bounded. For every $n \in \mathbb{N}$ there exists $j_{0} \in\{1, \ldots, n N\}$ such that

$$
\left\|D_{n}\right\|_{2}=\lambda_{1}\left(D_{n}\right)=\left[D_{n}\right]_{j_{0}, j_{0}}=\frac{\left\|D_{n} e_{j_{0}}\right\|_{F}}{\left\|e_{j_{0}}\right\|_{F}} \leq \frac{\left\|\left(T_{n}(F)\right)^{-1} L_{n} e_{j_{0}}\right\|_{F}}{\left\|e_{j_{0}}\right\|_{F}} \leq\left\|\left(T_{n}(F)\right)^{-1} L_{n}\right\|_{2} \leq\left\|\left(T_{n}(F)\right)^{-1}\right\|_{2}\left\|L_{n}\right\|_{2}
$$

where $e_{j_{0}}$ is the $n N$-dimensional (column) vector whose entries are given by $\left[e_{j_{0}}\right]_{j, 1}=\delta_{j, j_{0}}, j \in$ $\{1, \ldots, n N\}$, with $\delta$ being the Kronecker delta. Thus, $\left\{\left\|D_{n}\right\|_{2}\right\}$ is bounded.

Observe that Equation (3) shows that the sequence $\left\{\frac{\left\|L_{n}-T_{n}(F)\right\|_{F}}{\sqrt{n}}\right\}$ converges to zero at least as fast as the sequence $\left\{\frac{\left\|A_{n}-T_{n}(F)\left(T_{n}(F)\right)^{*}\right\|_{F}}{\sqrt{n}}\right\}$ does.

Equation (2) generalizes ([3], Section 6.3). Specifically, in ([3], Section 6.3) Gray proved Equation (2), but only for the special case in which $N=1, F$ is in the Wiener class, and $\left\{A_{n}\right\}=\left\{T_{n}\left(F F^{*}\right)\right\}$ (or equivalently, $\left\{A_{n}\right\}=\left\{T_{n}\left(|F|^{2}\right)\right\}$ ). It should be mentioned that unlike here, the convergence speed of $\left\{\frac{\left\|L_{n}-T_{n}(F)\right\|_{F}}{\sqrt{n}}\right\}$ was not given in ([3], Section 6.3) for the special case there studied.

\section{Applications of the Periodogram Method in Parameter Estimation}

Using Theorems 1 and 2 we give in this section a parameter estimation method for perturbed VAR processes and another for perturbed VMA processes. These methods can be applied in any real-world problem where the random process involved is modeled as a VAR process or as a VMA process, e.g., in damage detection for aeronautical structures or in MIMO channel identification.

\subsection{Parameter Estimation Method for Perturbed VAR Processes}

We begin by reviewing the concept of VAR process.

Definition 1. A zero-mean random $N$-dimensional vector process $\left\{x_{n}\right\}$ is said to be a VAR process if

$$
x_{n}=w_{n}-\sum_{k=1}^{n-1} \mathrm{~F}_{-k} x_{n-k} \quad \forall n \in \mathbb{N},
$$

where $\mathrm{F}_{-k} \in \mathbb{C}^{N \times N}$ for all $k \in \mathbb{N}$, and $\left\{w_{n}\right\}$ is a zero-mean random $N$-dimensional vector process whose sequence of correlation matrices is given by $\left\{E\left(w_{n: 1} w_{n: 1}^{*}\right)\right\}=\left\{T_{n}(\Lambda)\right\}$ with $\Lambda$ being an $N \times N$ positive definite matrix. If there exists $p \in \mathbb{N}$ such that $\mathrm{F}_{-k}=0_{N \times N}$ for all $k>p$, then $\left\{x_{n}\right\}$ is called a VAR process of (finite) order $p$ or a $\operatorname{VAR}(p)$ process. 
Let $\left\{x_{n}\right\}$ be as in Definition 1. Assume that $\left\{F_{k}\right\}_{k \in \mathbb{Z}}$, with $F_{0}=I_{N}$ and $F_{k}=0_{N \times N}$ for all $k \in \mathbb{N}$, is the sequence of Fourier coefficients of a continuous $2 \pi$-periodic function $F: \mathbb{R} \rightarrow \mathbb{C}^{N \times N}$. From Equation (8) we can obtain (see, e.g., ([12], Equation (20)))

$$
\left(E\left(x_{n: 1} x_{n: 1}^{*}\right)\right)^{-1}=T_{n}\left(F^{*}\right) T_{n}\left(\Lambda^{-1}\right) T_{n}(F) \quad \forall n \in \mathbb{N} .
$$

If $\Lambda^{-1}=L_{\Lambda^{-1}} L_{\Lambda^{-1}}^{*}$ is the Cholesky decomposition of the positive definite matrix $\Lambda^{-1}$, then

$$
\left(E\left(x_{n: 1} x_{n: 1}^{*}\right)\right)^{-1}=T_{n}\left(F^{*} L_{\Lambda^{-1}}\right)\left(T_{n}\left(F^{*} L_{\Lambda^{-1}}\right)\right)^{*}
$$

is the Cholesky decomposition of the positive definite matrix $\left(E\left(x_{n: 1} x_{n: 1}^{*}\right)\right)^{-1}$ for all $n \in \mathbb{N}$, since

$$
\begin{aligned}
\left(E\left(x_{n: 1} x_{n: 1}^{*}\right)\right)^{-1} & =T_{n}\left(F^{*}\right) T_{n}\left(L_{\Lambda^{-1}} L_{\Lambda^{-1}}^{*}\right) T_{n}(F)=T_{n}\left(F^{*}\right) T_{n}\left(L_{\Lambda^{-1}}\right) T_{n}\left(L_{\Lambda^{-1}}^{*}\right) T_{n}(F) \\
& =T_{n}\left(F^{*} L_{\Lambda^{-1}}\right) T_{n}\left(L_{\Lambda^{-1}}^{*} F\right)=T_{n}\left(F^{*} L_{\Lambda^{-1}}\right) T_{n}\left(\left(F^{*} L_{\Lambda^{-1}}\right)^{*}\right) \quad \forall n \in \mathbb{N} .
\end{aligned}
$$

Observe that if we know the correlation matrix $E\left(x_{n: 1} x_{n: 1}^{*}\right)$ for certain $n \in \mathbb{N}$, then the Cholesky decomposition of $\left(E\left(x_{n: 1} x_{n: 1}^{*}\right)\right)^{-1}$ provides $\Lambda$ and the parameters $\mathrm{F}_{-1}, \ldots, \mathrm{F}_{1-n}$ of the VAR process, because

$$
T_{n}\left(F^{*} L_{\Lambda^{-1}}\right)=\left(\begin{array}{ccccc}
L_{\Lambda^{-1}} & 0_{N \times N} & 0_{N \times N} & \cdots & 0_{N \times N} \\
F_{-1}^{*} L_{\Lambda^{-1}} & L_{\Lambda^{-1}} & 0_{N \times N} & \cdots & 0_{N \times N} \\
\mathrm{~F}_{-2}^{*} L_{\Lambda^{-1}} & \mathrm{~F}_{-1}^{*} L_{\Lambda^{-1}} & L_{\Lambda^{-1}} & \cdots & 0_{N \times N} \\
\vdots & \vdots & \vdots & \ddots & \vdots \\
\mathrm{F}_{1-n}^{*} L_{\Lambda^{-1}} & \mathrm{~F}_{2-n}^{*} L_{\Lambda^{-1}} & \mathrm{~F}_{3-n}^{*} L_{\Lambda^{-1}} & \cdots & L_{\Lambda^{-1}}
\end{array}\right) .
$$

However, in practice what we usually know is a perturbed version $\left\{A_{n}\right\}$ of the sequence of correlation matrices $\left\{E\left(x_{n: 1} x_{n: 1}^{*}\right)\right\}$ of the process. The following theorem allows us to estimate $\Lambda$ and the parameters $\left\{\mathrm{F}_{-k}\right\}_{k \in \mathbb{N}}$ of the VAR process from the Cholesky decomposition of the matrices of the sequence $\left\{A_{n}^{-1}\right\}$, when $\left\{A_{n}\right\} \sim\left\{E\left(x_{n: 1} x_{n: 1}^{*}\right)\right\}$.

Theorem 3. Let $\left\{x_{n}\right\}$ be as in Definition 1. Assume that $\left\{\mathrm{F}_{k}\right\}_{k \in \mathbb{Z}}$, with $\mathrm{F}_{0}=I_{N}$ and $\mathrm{F}_{k}=0_{N \times N}$ for all $k \in \mathbb{N}$, is the sequence of Fourier coefficients of a continuous $2 \pi$-periodic function $F: \mathbb{R} \rightarrow \mathbb{C}^{N \times N}$. Suppose that $A_{n}$ is an $n N \times n N$ positive definite matrix for all $n \in \mathbb{N}$ satisfying that $\left\{A_{n}\right\}$ is stable and $\left\{A_{n}\right\} \sim\left\{E\left(x_{n: 1} x_{n: 1}^{*}\right)\right\}$. Let $A_{n}^{-1}=L_{n} L_{n}^{*}$ be the Cholesky decomposition of $A_{n}^{-1}$ for all $n \in \mathbb{N}$. Then

$$
\lim _{n \rightarrow \infty} \frac{1}{2 \pi} \int_{0}^{2 \pi}\left\|\widehat{P}_{L_{n}}(\omega)-(F(\omega))^{*} L_{\Lambda^{-1}}\right\|_{F}^{2} d \omega=0
$$

and

$$
\left\|\frac{1}{2 \pi} \int_{0}^{2 \pi} \mathrm{e}^{-k \omega \mathrm{i}} \widehat{P}_{L_{n}}(\omega) d \omega-\mathrm{F}_{-k}^{*} L_{\Lambda^{-1}}\right\|_{F}^{2} \leq \frac{1}{2 \pi} \int_{0}^{2 \pi}\left\|\widehat{P}_{L_{n}}(\omega)-(F(\omega))^{*} L_{\Lambda^{-1}}\right\|_{F}^{2} d \omega
$$

for all $n \in \mathbb{N}$ and $k \in\{0,1, \ldots, n-1\}$, where $\Lambda^{-1}=L_{\Lambda^{-1}} L_{\Lambda^{-1}}$ is the Cholesky decomposition of $\Lambda^{-1}$. Moreover, if $\left\{x_{n}\right\}$ is of finite order there exist $K_{1}, K_{2} \in[0, \infty)$ such that

$$
\sqrt{\frac{1}{2 \pi} \int_{0}^{2 \pi}\left\|\widehat{P}_{L_{n}}(\omega)-(F(\omega))^{*} L_{\Lambda^{-1}}\right\|_{F}^{2} d \omega} \leq K_{1} \frac{\left\|A_{n}-E\left(x_{n: 1} x_{n: 1}^{*}\right)\right\|_{F}}{\sqrt{n}}+\frac{K_{2}}{\sqrt{n}} \quad \forall n \in \mathbb{N} .
$$

Proof. Since $A_{n}$ is positive definite matrix for all $n \in \mathbb{N}, A_{n}^{-1}$ is positive definite matrix for all $n \in \mathbb{N}$. From ([12], Equation (20)) and ([5], Lemma 4.2) we have

$$
\left\|\left(E\left(x_{n: 1} x_{n: 1}^{*}\right)\right)^{-1}\right\|_{2}=\left\|T_{n}\left(F^{*}\right) T_{n}\left(\Lambda^{-1}\right) T_{n}(F)\right\|_{2} \leq\left\|T_{n}\left(F^{*}\right)\right\|_{2}\left\|T_{n}\left(\Lambda^{-1}\right)\right\|_{2}\left\|T_{n}(F)\right\|_{2}
$$




$$
=\left\|\left(T_{n}(F)\right)^{*}\right\|_{2}\left\|\Lambda^{-1}\right\|_{2}\left\|T_{n}(F)\right\|_{2}=\left\|\Lambda^{-1}\right\|_{2}\left\|T_{n}(F)\right\|_{2}^{2} \quad \forall n \in \mathbb{N} .
$$

Hence, as $\left\{\left\|T_{n}(F)\right\|_{2}\right\}$ is bounded (see, e.g., ([5], Theorem 4.3) or ([9], Corollary 4.2)), $\left\{\left\|\left(E\left(x_{n: 1} x_{n: 1}^{*}\right)\right)^{-1}\right\|_{2}\right\}$ is also bounded. Consequently, applying ([13], Lemma A1) and Equation (9) yields

$$
\left\{A_{n}^{-1}\right\} \sim\left\{\left(E\left(x_{n: 1} x_{n: 1}^{*}\right)\right)^{-1}\right\}=\left\{T_{n}\left(F^{*} L_{\Lambda^{-1}}\right)\left(T_{n}\left(F^{*} L_{\Lambda^{-1}}\right)\right)^{*}\right\}
$$

As $\left\{\left\|A_{n}\right\|_{2}\right\}$ and $\left\{\left\|E\left(x_{n: 1} x_{n: 1}^{*}\right)\right\|_{2}\right\}$ are bounded, the sequences

$$
\left\{\left\|L_{n}^{-1}\right\|_{2}\right\}=\left\{\sqrt{\lambda_{1}\left(\left(L_{n}^{-1}\right)^{*} L_{n}^{-1}\right)}\right\}=\left\{\sqrt{\left\|\left(L_{n}^{-1}\right)^{*} L_{n}^{-1}\right\|_{2}}\right\}=\left\{\sqrt{\left\|\left(L_{n} L_{n}^{*}\right)^{-1}\right\|_{2}}\right\}=\left\{\sqrt{\left\|A_{n}\right\|_{2}}\right\}
$$

and

$$
\left\{\left\|\left(T_{n}\left(F^{*} L_{\Lambda^{-1}}\right)\right)^{-1}\right\|_{2}\right\}=\left\{\sqrt{\left\|\left(T_{n}\left(F^{*} L_{\Lambda^{-1}}\right)\left(T_{n}\left(F^{*} L_{\Lambda^{-1}}\right)\right)^{*}\right)^{-1}\right\|_{2}}\right\}=\left\{\sqrt{\left\|E\left(x_{n: 1} x_{n: 1}^{*}\right)\right\|_{2}}\right\}
$$

are also bounded. Thus, from Theorem 2 we have that $\left\{L_{n}\right\} \sim\left\{T_{n}\left(F^{*} L_{\Lambda^{-1}}\right)\right\}$ and that there exists $K \in[0, \infty)$ such that

$$
\frac{\left\|L_{n}-T_{n}\left(F^{*} L_{\Lambda^{-1}}\right)\right\|_{F}}{\sqrt{n}} \leq K \frac{\left\|A_{n}^{-1}-T_{n}\left(F^{*} L_{\Lambda^{-1}}\right)\left(T_{n}\left(F^{*} L_{\Lambda^{-1}}\right)\right)^{*}\right\|_{F}}{\sqrt{n}}=K \frac{\left\|A_{n}^{-1}-\left(E\left(x_{n: 1} x_{n: 1}^{*}\right)\right)^{-1}\right\|_{F}}{\sqrt{n}}
$$

for all $n \in \mathbb{N}$. Hence, applying Theorem 1 we conclude that Equation (11) holds.

Applying the Schwarz inequality (see, e.g., ([14], p. 139) yields

$$
\begin{aligned}
& \left\|\frac{1}{2 \pi} \int_{0}^{2 \pi} \mathrm{e}^{-k \omega \mathrm{i}} \widehat{P}_{L_{n}}(\omega) d \omega-\mathrm{F}_{-k}^{*} L_{\Lambda^{-1}}\right\|_{F}=\left\|\frac{1}{2 \pi} \int_{0}^{2 \pi} \mathrm{e}^{-k \omega \mathrm{i}} \widehat{P}_{L_{n}}(\omega) d \omega-\frac{1}{2 \pi} \int_{0}^{2 \pi} \mathrm{e}^{-k \omega \mathrm{i}}(F(\omega))^{*} d \omega L_{\Lambda^{-1}}\right\|_{F} \\
& =\frac{1}{2 \pi}\left\|\int_{0}^{2 \pi} \mathrm{e}^{-k \omega \mathrm{i}}\left(\widehat{P}_{L_{n}}(\omega)-(F(\omega))^{*} L_{\Lambda^{-1}}\right) d \omega\right\|_{F} \\
& =\frac{1}{2 \pi} \sqrt{\sum_{r, s=1}^{N}\left|\left[\int_{0}^{2 \pi} \mathrm{e}^{-k \omega \mathrm{i}}\left(\widehat{P}_{L_{n}}(\omega)-(F(\omega))^{*} L_{\Lambda^{-1}}\right) d \omega\right]_{r, s}\right|^{2}} \\
& =\frac{1}{2 \pi} \sqrt{\sum_{r, s=1}^{N}\left|\int_{0}^{2 \pi} \mathrm{e}^{-k \omega \mathrm{i}}\left[\widehat{P}_{L_{n}}(\omega)-(F(\omega))^{*} L_{\Lambda^{-1}}\right]_{r, s} d \omega\right|^{2}} \\
& \leq \frac{1}{2 \pi} \sqrt{\sum_{r, s=1}^{N} 2 \pi \int_{0}^{2 \pi}\left|\mathrm{e}^{-k \omega \mathrm{i}}\left[\widehat{P}_{L_{n}}(\omega)-(F(\omega))^{*} L_{\Lambda^{-1}}\right]_{r, s}\right|^{2} d \omega} \\
& =\sqrt{\frac{1}{2 \pi} \int_{0}^{2 \pi} \sum_{r, s=1}^{N}\left|\mathrm{e}^{-k \omega \mathrm{i}}\right|^{2}\left|\left[\widehat{P}_{L_{n}}(\omega)-(F(\omega))^{*} L_{\Lambda^{-1}}\right]_{r, s}\right|^{2} d \omega} \\
& =\sqrt{\frac{1}{2 \pi} \int_{0}^{2 \pi} \sum_{r, s=1}^{N}\left|\left[\widehat{P}_{L_{n}}(\omega)-(F(\omega))^{*} L_{\Lambda^{-1}}\right]_{r, s}\right|^{2} d \omega} \\
& =\sqrt{\frac{1}{2 \pi} \int_{0}^{2 \pi}\left\|\widehat{P}_{L_{n}}(\omega)-(F(\omega))^{*} L_{\Lambda^{-1}}\right\|_{F}^{2} d \omega}
\end{aligned}
$$

for all $n \in \mathbb{N}$ and $k \in \mathbb{Z}$. 
Moreover, if $\left\{x_{n}\right\}$ is of finite order from Theorem 1 there exists $K_{2} \in[0, \infty)$ such that

$$
\begin{aligned}
& \sqrt{\frac{1}{2 \pi} \int_{0}^{2 \pi}\left\|\widehat{P}_{L_{n}}(\omega)-(F(\omega))^{*} L_{\Lambda^{-1}}\right\|_{F}^{2} d \omega} \\
& \leq \frac{\left\|L_{n}-T_{n}\left(F^{*} L_{\Lambda^{-1}}\right)\right\|_{F}}{\sqrt{n}}+\frac{K_{2}}{\sqrt{n}} \\
& \leq K \frac{\left\|A_{n}^{-1}-\left(E\left(x_{n: 1} x_{n: 1}^{*}\right)\right)^{-1}\right\|_{F}}{\sqrt{n}}+\frac{K_{2}}{\sqrt{n}} \\
& =K \frac{\left\|\left(E\left(x_{n: 1} x_{n: 1}^{*}\right)\right)^{-1}-A_{n}^{-1}\right\|_{F}}{\sqrt{n}}+\frac{K_{2}}{\sqrt{n}} \\
& =K \frac{\left\|\left(E\left(x_{n: 1} x_{n: 1}^{*}\right)\right)^{-1}\left(A_{n}-E\left(x_{n: 1} x_{n: 1}^{*}\right)\right) A_{n}^{-1}\right\|_{F}}{\sqrt{n}}+\frac{K_{2}}{\sqrt{n}} \\
& \leq K\left\|\left(E\left(x_{n: 1} x_{n: 1}^{*}\right)\right)^{-1}\right\|_{2} \frac{\left\|\left(A_{n}-E\left(x_{n: 1} x_{n: 1}^{*}\right)\right) A_{n}^{-1}\right\|_{F}}{\sqrt{n}}+\frac{K_{2}}{\sqrt{n}} \\
& \leq K\left\|\left(E\left(x_{n: 1} x_{n: 1}^{*}\right)\right)^{-1}\right\|_{2}\left\|A_{n}^{-1}\right\|_{2} \frac{\left\|A_{n}-E\left(x_{n: 1} x_{n: 1}^{*}\right)\right\|_{F}}{\sqrt{n}}+\frac{K_{2}}{\sqrt{n}} \quad \forall n \in \mathbb{N} .
\end{aligned}
$$

If we know $A_{n}$ for certain $n \in \mathbb{N}$, Theorem 3 provides an estimation of the block entry $\mathrm{F}_{-k}^{*} L_{\Lambda^{-1}}$ of the matrix $T_{n}\left(F^{*} L_{\Lambda^{-1}}\right)$ in Equation (10) given by

$$
\begin{aligned}
& \frac{1}{2 \pi} \int_{0}^{2 \pi} \mathrm{e}^{-k \omega \mathrm{i}} \widehat{P}_{L_{n}}(\omega) d \omega \\
& =\frac{1}{2 \pi} \int_{0}^{2 \pi} \mathrm{e}^{-k \omega \mathrm{i}} \sum_{h=1}^{n} \chi_{\left[\frac{2 \pi(h-1)}{n}, \frac{2 \pi h}{n}\right)}(\omega)\left[\left(V_{n} \otimes I_{N}\right)^{*} L_{n}\left(V_{n} \otimes I_{N}\right)\right]_{h, h} d \omega \\
& =\frac{1}{2 \pi} \sum_{h=1}^{n} \int_{0}^{2 \pi} \chi_{\left[\frac{2 \pi(h-1)}{n}, \frac{2 \pi h}{n}\right)}(\omega) \mathrm{e}^{-k \omega \mathrm{i}} d \omega\left[\left(V_{n} \otimes I_{N}\right)^{*} L_{n}\left(V_{n} \otimes I_{N}\right)\right]_{h, h} \\
& =\frac{1}{2 \pi} \sum_{h=1}^{n} \int_{\frac{2 \pi(h-1)}{n}}^{\frac{2 \pi h}{n}} \mathrm{e}^{-k \omega \mathrm{i}} d \omega\left[\left(V_{n} \otimes I_{N}\right)^{*} L_{n}\left(V_{n} \otimes I_{N}\right)\right]_{h, h} \\
& = \begin{cases}\frac{1}{n} \sum_{h=1}^{n}\left[\left(V_{n} \otimes I_{N}\right)^{*} L_{n}\left(V_{n} \otimes I_{N}\right)\right]_{h, h} & \text { if } k=0, \\
\frac{\mathrm{i}}{2 \pi k} \sum_{h=1}^{n}\left(\mathrm{e}^{-k \frac{2 \pi h}{n} \mathrm{i}}-\mathrm{e}^{-k \frac{2 \pi(h-1)}{n} \mathrm{i}}\right)\left[\left(V_{n} \otimes I_{N}\right)^{*} L_{n}\left(V_{n} \otimes I_{N}\right)\right]_{h, h} & \text { if } k \in\{1, \ldots, n-1\} .\end{cases}
\end{aligned}
$$

Therefore, if we know $A_{n}$ for certain $n \in \mathbb{N}$, Theorem 3 allows us to estimate $\Lambda$ and the parameters $\mathrm{F}_{-1}, \ldots, \mathrm{F}_{1-n}$ of the VAR process as follows

$$
\widehat{\Lambda}(n)=\left(\left(\frac{1}{2 \pi} \int_{0}^{2 \pi} \widehat{P}_{L_{n}}(\omega) d \omega\right)\left(\frac{1}{2 \pi} \int_{0}^{2 \pi} \widehat{P}_{L_{n}}(\omega) d \omega\right)^{*}\right)^{-1}
$$

and

$$
\widehat{\mathrm{F}}_{-k}(n)=\left(\left(\frac{1}{2 \pi} \int_{0}^{2 \pi} \mathrm{e}^{-k \omega \mathrm{i}} \widehat{P}_{L_{n}}(\omega) d \omega\right)\left(\frac{1}{2 \pi} \int_{0}^{2 \pi} \widehat{P}_{L_{n}}(\omega) d \omega\right)^{-1}\right)^{*} \quad \forall k \in\{1, \ldots, n-1\} .
$$


Example 1. We consider the zero-mean 2-dimensional VAR(1) process $\left\{x_{n}\right\}$ in ([15], Example 2.3), where

$$
\Lambda=\left(\begin{array}{ll}
4 & 1 \\
1 & 2
\end{array}\right)
$$

and

$$
F_{-1}=\left(\begin{array}{cc}
-0.8 & -0.7 \\
0.4 & -0.6
\end{array}\right)
$$

Figure 1 shows the squared error made when $\Lambda$ and $F_{-1}$ are estimated from the perturbed VAR(1) process whose sequence of correlation matrices is

$$
\left\{A_{n}\right\}=\left\{E\left(x_{n: 1} x_{n: 1}^{*}\right)+\left(\begin{array}{cc}
0_{2 n-2 \times 2 n-2} & 0_{2 n-2 \times 2} \\
0_{2 \times 2 n-2} & I_{2}
\end{array}\right)\right\} .
$$

Observe that this perturbed process has been generated by corrupting the VAR(1) process in ([15], Example 2.3) by an impulse at $n=1$.

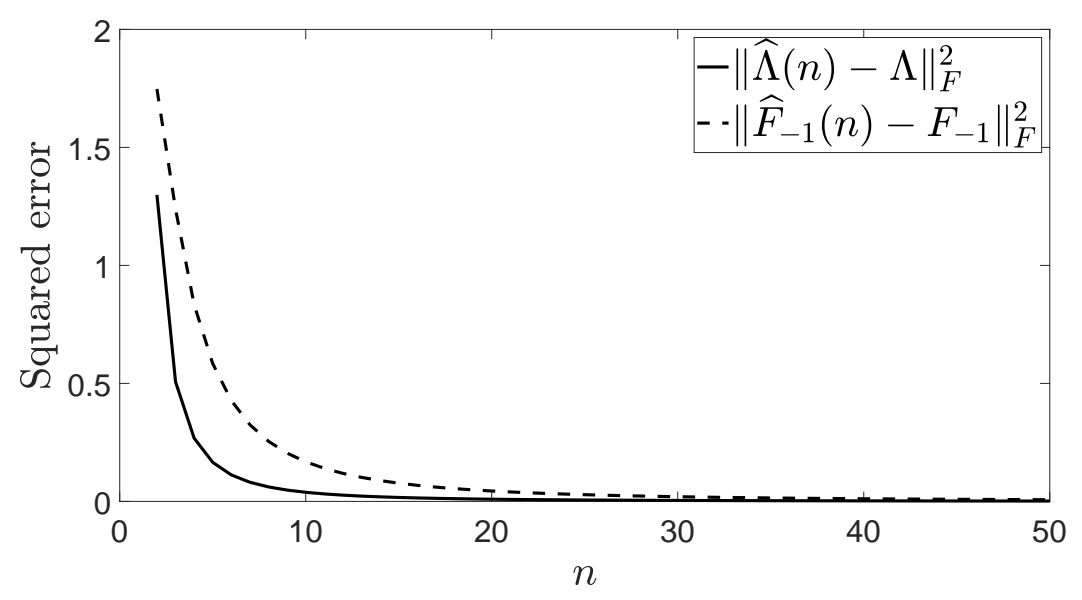

Figure 1. Squared error made when $\Lambda$ and $F_{-1}$ are estimated by $\widehat{\Lambda}(n)$ and $\widehat{F}_{-1}(n)$, respectively.

\subsection{Parameter Estimation Method for Perturbed VMA Processes}

We begin by reviewing the concept of VMA process.

Definition 2. A zero-mean random $N$-dimensional vector process $\left\{x_{n}\right\}$ is said to be a VMA process if

$$
x_{n}=w_{n}+\sum_{k=1}^{n-1} \mathrm{G}_{k} w_{n-k} \quad \forall n \in \mathbb{N},
$$

where $\mathrm{G}_{k} \in \mathbb{C}^{N \times N}$ for all $k \in \mathbb{N}$, and $\left\{w_{n}\right\}$ is a zero-mean random $N$-dimensional vector process whose sequence of correlation matrices is given by $\left\{E\left(w_{1: n} w_{1: n}^{*}\right)\right\}=\left\{T_{n}(\Lambda)\right\}$ with $\Lambda$ being an $N \times N$ positive definite matrix and

$$
w_{1: n}=\left(\begin{array}{c}
w_{1} \\
\vdots \\
w_{n}
\end{array}\right) \quad \forall n \in \mathbb{N}
$$

If there exists $q \in \mathbb{N}$ such that $\mathrm{G}_{k}=0_{N \times N}$ for all $k>q$, then $\left\{x_{n}\right\}$ is called a VMA process of (finite) order q or a $\operatorname{VMA}(q)$ process. 
Let $\left\{x_{n}\right\}$ be as in Definition 2. Assume that $\left\{G_{k}\right\}_{k \in \mathbb{Z}}$, with $G_{0}=I_{N}$ and $G_{-k}=0_{N \times N}$ for all $k \in \mathbb{N}$, is the sequence of Fourier coefficients of a continuous $2 \pi$-periodic function $G: \mathbb{R} \rightarrow \mathbb{C}^{N \times N}$. Since Equation (12) can be rewritten as

$$
x_{n}=\left(\begin{array}{llll}
G_{n-1} & \cdots & G_{1} & I_{N}
\end{array}\right) w_{1: n} \quad \forall n \in \mathbb{N},
$$

we have

$$
x_{1: n}=\left(\begin{array}{ccccc}
I_{N} & 0_{N \times N} & 0_{N \times N} & \cdots & 0_{N \times N} \\
G_{1} & I_{N} & 0_{N \times N} & \cdots & 0_{N \times N} \\
G_{2} & G_{1} & I_{N} & \cdots & 0_{N \times N} \\
\vdots & \vdots & \vdots & \ddots & \vdots \\
G_{n-1} & G_{n-2} & G_{n-3} & \cdots & I_{N}
\end{array}\right) w_{1: n}=T_{n}(G) w_{1: n} \quad \forall n \in \mathbb{N}
$$

and consequently,

$$
\begin{aligned}
\left\{E\left(x_{1: n} x_{1: n}^{*}\right)\right\} & =\left\{E\left(T_{n}(G) w_{1: n} w_{1: n}^{*}\left(T_{n}(G)\right)^{*}\right)\right\} \\
& =\left\{T_{n}(G) E\left(w_{1: n} w_{1: n}^{*}\right)\left(T_{n}(G)\right)^{*}\right\}=\left\{T_{n}(G) T_{n}(\Lambda)\left(T_{n}(G)\right)^{*}\right\} .
\end{aligned}
$$

If $\Lambda=L_{\Lambda} L_{\Lambda}^{*}$ is the Cholesky decomposition of $\Lambda$, then

$$
E\left(x_{1: n} x_{1: n}^{*}\right)=T_{n}\left(G L_{\Lambda}\right)\left(T_{n}\left(G L_{\Lambda}\right)\right)^{*}
$$

is the Cholesky decomposition of the positive definite matrix $E\left(x_{1: n} x_{1: n}^{*}\right)$ for all $n \in \mathbb{N}$, because

$$
\begin{aligned}
E\left(x_{1: n} x_{1: n}^{*}\right) & =T_{n}(G) T_{n}\left(L_{\Lambda} L_{\Lambda}^{*}\right)\left(T_{n}(G)\right)^{*}=T_{n}(G) T_{n}\left(L_{\Lambda}\right) T_{n}\left(L_{\Lambda}^{*}\right)\left(T_{n}(G)\right)^{*} \\
& =T_{n}\left(G L_{\Lambda}\right)\left(T_{n}\left(L_{\Lambda}\right)\right)^{*}\left(T_{n}(G)\right)^{*}=T_{n}\left(G L_{\Lambda}\right)\left(T_{n}(G) T_{n}\left(L_{\Lambda}\right)\right)^{*} \quad \forall n \in \mathbb{N} .
\end{aligned}
$$

Observe that if we know the correlation matrix $E\left(x_{1: n} x_{1: n}^{*}\right)$ for certain $n \in \mathbb{N}$, then its Cholesky decomposition provides $\Lambda$ and the parameters $G_{1}, \ldots, G_{n-1}$ of the VMA process, since

$$
T_{n}\left(G L_{\Lambda}\right)=\left(\begin{array}{ccccc}
L_{\Lambda} & 0_{N \times N} & 0_{N \times N} & \cdots & 0_{N \times N} \\
G_{1} L_{\Lambda} & L_{\Lambda} & 0_{N \times N} & \cdots & 0_{N \times N} \\
G_{2} L_{\Lambda} & G_{1} L_{\Lambda} & L_{\Lambda} & \cdots & 0_{N \times N} \\
\vdots & \vdots & \vdots & \ddots & \vdots \\
G_{n-1} L_{\Lambda} & G_{n-2} L_{\Lambda} & G_{n-3} L_{\Lambda} & \cdots & L_{\Lambda}
\end{array}\right) .
$$

However, in practice what we usually know is a perturbed version $\left\{A_{n}\right\}$ of the sequence of correlation matrices $\left\{E\left(x_{1: n} x_{1: n}^{*}\right)\right\}$ of the process. The following theorem allows us to estimate $\Lambda$ and the parameters $\left\{G_{k}\right\}_{k \in \mathbb{N}}$ of the VMA process from the Cholesky decomposition of the matrices of the sequence $\left\{A_{n}\right\}$, when $\left\{A_{n}\right\} \sim\left\{E\left(x_{1: n} x_{1: n}^{*}\right)\right\}$.

Theorem 4. Let $\left\{x_{n}\right\}$ be as in Definition 2. Assume that $\left\{\mathrm{G}_{k}\right\}_{k \in \mathbb{Z}}$, with $\mathrm{G}_{0}=I_{N}$ and $\mathrm{G}_{-k}=0_{N \times N}$ for all $k \in \mathbb{N}$, is the sequence of Fourier coefficients of a continuous $2 \pi$-periodic function $G: \mathbb{R} \rightarrow \mathbb{C}^{N \times N}$. Suppose that $A_{n}$ is an $n N \times n N$ positive definite matrix for all $n \in \mathbb{N}$ satisfying that $\left\{A_{n}\right\}$ is stable and $\left\{A_{n}\right\} \sim\left\{E\left(x_{1: n} x_{1: n}^{*}\right)\right\}$. Let $A_{n}=L_{n} L_{n}^{*}$ be the Cholesky decomposition of $A_{n}$ for all $n \in \mathbb{N}$. If $\left\{E\left(x_{1: n} x_{1: n}^{*}\right)\right\}$ is stable then

$$
\lim _{n \rightarrow \infty} \frac{1}{2 \pi} \int_{0}^{2 \pi}\left\|\widehat{P}_{L_{n}}(\omega)-G(\omega) L_{\Lambda}\right\|_{F}^{2} d \omega=0,
$$

and

$$
\left\|\frac{1}{2 \pi} \int_{0}^{2 \pi} \mathrm{e}^{-k \omega \mathrm{i}} \widehat{P}_{L_{n}}(\omega) d \omega-\mathrm{G}_{k} L_{\Lambda}\right\|_{F}^{2} \leq \frac{1}{2 \pi} \int_{0}^{2 \pi}\left\|\widehat{P}_{L_{n}}(\omega)-G(\omega) L_{\Lambda}\right\|_{F}^{2} d \omega
$$


for all $n \in \mathbb{N}$ and $k \in\{0,1, \ldots, n-1\}$, where $\Lambda=L_{\Lambda} L_{\Lambda}^{*}$ is the Cholesky decomposition of $\Lambda$. Moreover, if $\left\{x_{n}\right\}$ is of finite order there exist $K_{1}, K_{2} \in[0, \infty)$ such that

$$
\sqrt{\frac{1}{2 \pi} \int_{0}^{2 \pi}\left\|\widehat{P}_{L_{n}}(\omega)-G(\omega) L_{\Lambda}\right\|_{F}^{2} d \omega} \leq K_{1} \frac{\left\|A_{n}-E\left(x_{1: n} x_{1: n}^{*}\right)\right\|_{F}}{\sqrt{n}}+\frac{K_{2}}{\sqrt{n}} \quad \forall n \in \mathbb{N} .
$$

Proof. From Equation (14) we have $\left\{A_{n}\right\} \sim\left\{E\left(x_{1: n} x_{1: n}^{*}\right)\right\}=\left\{T_{n}\left(G L_{\Lambda}\right)\left(T_{n}\left(G L_{\Lambda}\right)\right)^{*}\right\}$. As $\left\{\left\|A_{n}^{-1}\right\|_{2}\right\}$ and $\left\{\left\|\left(E\left(x_{1: n} x_{1: n}^{*}\right)\right)^{-1}\right\|_{2}\right\}$ are bounded, the sequences

$$
\left\{\left\|L_{n}^{-1}\right\|_{2}\right\}=\left\{\sqrt{\lambda_{1}\left(\left(L_{n}^{-1}\right)^{*} L_{n}^{-1}\right)}\right\}=\left\{\sqrt{\left\|\left(L_{n}^{-1}\right)^{*} L_{n}^{-1}\right\|_{2}}\right\}=\left\{\sqrt{\left\|\left(L_{n} L_{n}^{*}\right)^{-1}\right\|_{2}}\right\}=\left\{\sqrt{\left\|A_{n}^{-1}\right\|_{2}}\right\}
$$

and

$$
\left\{\left\|\left(T_{n}\left(G L_{\Lambda}\right)\right)^{-1}\right\|_{2}\right\}=\left\{\sqrt{\left\|\left(T_{n}\left(G L_{\Lambda}\right)\left(T_{n}\left(G L_{\Lambda}\right)\right)^{*}\right)^{-1}\right\|_{2}}\right\}=\left\{\sqrt{\left\|\left(E\left(x_{1: n} x_{1: n}^{*}\right)\right)^{-1}\right\|_{2}}\right\}
$$

are also bounded. Consequently, from Theorem 2 we have that $\left\{L_{n}\right\} \sim\left\{T_{n}\left(G L_{\Lambda}\right)\right\}$ and that there exists $K_{1} \in[0, \infty)$ such that

$$
\frac{\left\|L_{n}-T_{n}\left(G L_{\Lambda}\right)\right\|_{F}}{\sqrt{n}} \leq K_{1} \frac{\left\|A_{n}-T_{n}\left(G L_{\Lambda}\right)\left(T_{n}\left(G L_{\Lambda}\right)\right)^{*}\right\|_{F}}{\sqrt{n}}=K_{1} \frac{\left\|A_{n}-E\left(x_{1: n} x_{1: n}^{*}\right)\right\|_{F}}{\sqrt{n}} \quad \forall n \in \mathbb{N} .
$$

Therefore, applying Theorem 1 we conclude that Equation (16) holds.

Applying the Schwarz inequality (see, e.g., ([14], p. 139)) yields

$$
\begin{aligned}
& \left\|\frac{1}{2 \pi} \int_{0}^{2 \pi} \mathrm{e}^{-k \omega \mathrm{i}} \widehat{P}_{L_{n}}(\omega) d \omega-\mathrm{G}_{k} L_{\Lambda}\right\|_{F}=\left\|\frac{1}{2 \pi} \int_{0}^{2 \pi} \mathrm{e}^{-k \omega \mathrm{i}} \widehat{P}_{L_{n}}(\omega) d \omega-\frac{1}{2 \pi} \int_{0}^{2 \pi} \mathrm{e}^{-k \omega \mathrm{i}} G(\omega) d \omega L_{\Lambda}\right\|_{F} \\
& =\frac{1}{2 \pi}\left\|\int_{0}^{2 \pi} \mathrm{e}^{-k \omega \mathrm{i}}\left(\widehat{P}_{L_{n}}(\omega)-G(\omega) L_{\Lambda}\right) d \omega\right\|_{F} \\
& =\frac{1}{2 \pi} \sqrt{\sum_{r, s=1}^{N}\left|\left[\int_{0}^{2 \pi} \mathrm{e}^{-k \omega \mathrm{i}}\left(\widehat{P}_{L_{n}}(\omega)-G(\omega) L_{\Lambda}\right) d \omega\right]_{r, s}\right|^{2}} \\
& =\frac{1}{2 \pi} \sqrt{\sum_{r, s=1}^{N}\left|\int_{0}^{2 \pi} \mathrm{e}^{-k \omega \mathrm{i}}\left[\widehat{P}_{L_{n}}(\omega)-G(\omega) L_{\Lambda}\right]_{r, s} d \omega\right|^{2}} \\
& \leq \frac{1}{2 \pi} \sqrt{\sum_{r, s=1}^{N} 2 \pi \int_{0}^{2 \pi}\left|\mathrm{e}^{-k \omega \mathrm{i}}\left[\widehat{P}_{L_{n}}(\omega)-G(\omega) L_{\Lambda}\right]_{r, s}\right|^{2} d \omega} \\
& =\sqrt{\frac{1}{2 \pi} \int_{0}^{2 \pi} \sum_{r, s=1}^{N}\left|\mathrm{e}^{-k \omega \mathrm{i}}\right|^{2}\left|\left[\widehat{P}_{L_{n}}(\omega)-G(\omega) L_{\Lambda}\right]_{r, s}\right|^{2} d \omega} \\
& =\sqrt{\frac{1}{2 \pi} \int_{0}^{2 \pi} \sum_{r, s=1}^{N}\left|\left[\widehat{P}_{L_{n}}(\omega)-G(\omega) L_{\Lambda}\right]_{r, s}\right|^{2} d \omega} \\
& =\sqrt{\frac{1}{2 \pi} \int_{0}^{2 \pi}\left\|\widehat{P}_{L_{n}}(\omega)-G(\omega) L_{\Lambda}\right\|_{F}^{2} d \omega}
\end{aligned}
$$

for all $n \in \mathbb{N}$ and $k \in \mathbb{Z}$. 
Moreover, if $\left\{x_{n}\right\}$ is of finite order from Theorem 1 there exists $K_{2} \in[0, \infty)$ such that

$$
\begin{aligned}
& \sqrt{\frac{1}{2 \pi} \int_{0}^{2 \pi}\left\|\widehat{P}_{L_{n}}(\omega)-G(\omega) L_{\Lambda}\right\|_{F}^{2} d \omega} \\
& \quad \leq \frac{\left\|L_{n}-T_{n}\left(G L_{\Lambda}\right)\right\|_{F}}{\sqrt{n}}+\frac{K_{2}}{\sqrt{n}} \leq K_{1} \frac{\left\|A_{n}-E\left(x_{1: n} x_{1: n}^{*}\right)\right\|_{F}}{\sqrt{n}}+\frac{K_{2}}{\sqrt{n}} \quad \forall n \in \mathbb{N} .
\end{aligned}
$$

If we know $A_{n}$ for certain $n \in \mathbb{N}$, Theorem 4 provides an estimation of the block entry $G_{k} L_{\Lambda}$ of the matrix $T_{n}\left(G L_{\Lambda}\right)$ in Equation (15) given by

$$
\begin{aligned}
& \frac{1}{2 \pi} \int_{0}^{2 \pi} \mathrm{e}^{-k \omega \mathrm{i}} \widehat{P}_{L_{n}}(\omega) d \omega \\
& = \begin{cases}\frac{1}{n} \sum_{h=1}^{n}\left[\left(V_{n} \otimes I_{N}\right)^{*} L_{n}\left(V_{n} \otimes I_{N}\right)\right]_{h, h} & \text { if } k=0, \\
\frac{1}{2 \pi k} \sum_{h=1}^{n}\left(\mathrm{e}^{-k \frac{2 \pi h}{n} \mathrm{i}}-\mathrm{e}^{-\frac{2 \pi(h-1)}{n} \mathrm{i}}\right)\left[\left(V_{n} \otimes I_{N}\right)^{*} L_{n}\left(V_{n} \otimes I_{N}\right)\right]_{h, h} & \text { if } k \in\{1, \ldots, n-1\} .\end{cases}
\end{aligned}
$$

Therefore, if we know $A_{n}$ for certain $n \in \mathbb{N}$, Theorem 4 allows us to estimate $\Lambda$ and the parameters $\mathrm{G}_{1}, \ldots, \mathrm{G}_{n-1}$ of the VMA process as follows

$$
\widehat{\Lambda}(n)=\left(\frac{1}{2 \pi} \int_{0}^{2 \pi} \widehat{P}_{L_{n}}(\omega) d \omega\right)\left(\frac{1}{2 \pi} \int_{0}^{2 \pi} \widehat{P}_{L_{n}}(\omega) d \omega\right)^{*}
$$

and

$$
\widehat{\mathrm{G}}_{k}(n)=\left(\frac{1}{2 \pi} \int_{0}^{2 \pi} \mathrm{e}^{-k \omega \mathrm{i}} \widehat{P}_{L_{n}}(\omega) d \omega\right)\left(\frac{1}{2 \pi} \int_{0}^{2 \pi} \widehat{P}_{L_{n}}(\omega) d \omega\right)^{-1} \quad \forall k \in\{1, \ldots, n-1\} .
$$

Example 2. We consider the zero-mean 2-dimensional VMA(1) process $\left\{x_{n}\right\}$ in ([15], Example 2.1), where

$$
\Lambda=\left(\begin{array}{ll}
4 & 1 \\
1 & 2
\end{array}\right)
$$

and

$$
G_{1}=\left(\begin{array}{cc}
-0.8 & -0.7 \\
0.4 & -0.6
\end{array}\right)
$$

Figure 2 shows the squared error made when $\Lambda$ and $G_{1}$ are estimated from the perturbed $V M A(1)$ process whose sequence of correlation matrices is

$$
\left\{A_{n}\right\}=\left\{E\left(x_{1: n} x_{1: n}^{*}\right)+\left(\begin{array}{cc}
I_{2} & 0_{2 \times 2 n-2} \\
0_{2 n-2 \times 2} & 0_{2 n-2 \times 2 n-2}
\end{array}\right)\right\} .
$$

Observe that this perturbed process has been generated by corrupting the VMA(1) process in ([15], Example 2.1) by an impulse at $n=1$. 


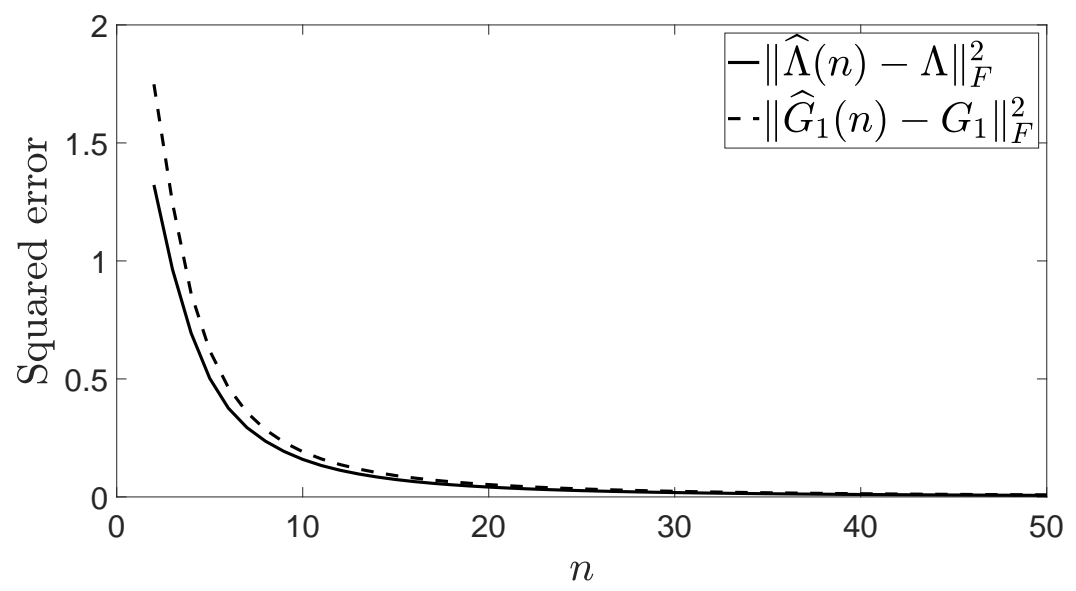

Figure 2. Squared error made when $\Lambda$ and $G_{1}$ are estimated by $\widehat{\Lambda}(n)$ and $\widehat{G}_{1}(n)$, respectively.

In [2], the periodogram method for perturbed block Toeplitz matrices was applied in spectral estimation. In Theorems 3 and 4, it has been also applied in parameter estimation for perturbed VAR processes and in parameter estimation for perturbed VMA processes, respectively. We finish the paper by showing that the periodogram method for perturbed block Toeplitz matrices can be applied in a fourth statistical signal processing problem, namely, in MIMO channel identification with perturbed additive WSS noise.

In [16], an asymptotic result on block Toeplitz matrices was applied in single-input multiple-output (SIMO) channel identification. We finish the paper by showing that Theorem 4 can be applied in MIMO channel identification when the number of channel inputs and the number of channel outputs are equal.

We consider a MIMO channel with a discrete-time causal infinite impulse response (IIR) filter and additive noise. Thus, the channel output process $\left\{y_{n}\right\}$ is of the form

$$
y_{n}=x_{n}+\epsilon_{n}=\sum_{k=0}^{n-1} \mathrm{G}_{k} w_{n-k}+\epsilon_{n} \quad \forall n \in \mathbb{N} \text {. }
$$

We assume that the filter tap $\mathrm{G}_{k} \in \mathbb{C}^{N \times N}$ for all $k \in \mathbb{N}$ and $\mathrm{G}_{0}=I_{N}$. We also assume that $\left\{G_{k}\right\}_{k \in \mathbb{Z}}$, with $G_{-k}=0_{N \times N}$ for all $k \in \mathbb{N}$, is the sequence of Fourier coefficients of a continuous $2 \pi$-periodic function $G: \mathbb{R} \rightarrow \mathbb{C}^{N \times N}$. We consider that the input process $\left\{w_{n}\right\}$ is a zero-mean WSS $N$-dimensional vector process with $\left\{E\left(w_{1: n} w_{1: n}^{*}\right)\right\}=\left\{T_{n}(\Lambda)\right\}$, where $\Lambda$ is an $N \times N$ positive definite matrix. We assume that the noise process $\left\{\epsilon_{n}\right\}$ is a zero-mean random $N$-dimensional vector process satisfying that there exists a continuous $2 \pi$-periodic function $Y: \mathbb{R} \rightarrow \mathbb{C}^{N \times N}$ such that $\left\{E\left(\epsilon_{1: n} \epsilon_{1: n}^{*}\right)\right\} \sim$ $\left\{T_{n}(Y)\right\}$. We also assume that the noise process is uncorrelated with the input process.

Suppose that $\left\{E\left(x_{1: n} x_{1: n}^{*}\right)\right\}$ is stable and $\left\{A_{n}\right\}=\left\{E\left(y_{1: n} y_{1: n}^{*}\right)-T_{n}(\mathrm{Y})\right\}$ is a stable sequence of positive definite matrices. To show that Theorem 4 can be here applied, we only need to prove that $\left\{A_{n}\right\} \sim\left\{E\left(x_{1: n} x_{1: n}^{*}\right)\right\}$.

From Equation (13) we obtain

$$
\left\|E\left(x_{1: n} x_{1: n}^{*}\right)\right\|_{2}=\left\|T_{n}(G) T_{n}(\Lambda)\left(T_{n}(G)\right)^{*}\right\|_{2} \leq\left\|T_{n}(G)\right\|_{2}\left\|T_{n}(\Lambda)\right\|_{2}\left\|\left(T_{n}(G)\right)^{*}\right\|_{2}=\|\Lambda\|_{2}\left\|T_{n}(G)\right\|_{2}^{2}
$$

for all $n \in \mathbb{N}$. Hence, as $\left\{\left\|T_{n}(G)\right\|_{2}\right\}$ is bounded (see, e.g., ([5], Theorem 4.3) or ([9], Corollary 4.2)), $\left\{\left\|E\left(x_{1: n} x_{1: n}^{*}\right)\right\|_{2}\right\}$ is also bounded and $\left\{E\left(x_{1: n} x_{1: n}^{*}\right)\right\} \sim\left\{E\left(x_{1: n} x_{1: n}^{*}\right)\right\}$. Since $\left\{\left\|-T_{n}(Y)\right\|_{2}\right\}=$ $\left\{\left\|T_{n}(\mathrm{Y})\right\|_{2}\right\}$ is bounded, $\left\{-T_{n}(\mathrm{Y})\right\} \sim\left\{-T_{n}(\mathrm{Y})\right\}$, and consequently, applying ([5], Lemma 3.1) yields $\left\{E\left(\epsilon_{1: n} \epsilon_{1: n}^{*}\right)-T_{n}(Y)\right\} \sim\left\{0_{n N \times n N}\right\}$. Therefore, from ([5], Lemma 3.1) we conclude that

$$
\left\{A_{n}\right\}=\left\{E\left(\left(x_{1: n}+\epsilon_{1: n}\right)\left(x_{1: n}+\epsilon_{1: n}\right)^{*}\right)-T_{n}(\mathrm{Y})\right\}
$$




$$
\begin{aligned}
& =\left\{E\left(x_{1: n} x_{1: n}^{*}\right)+E\left(x_{1: n} \epsilon_{1: n}^{*}\right)+E\left(\epsilon_{1: n} x_{1: n}^{*}\right)+E\left(\epsilon_{1: n} \epsilon_{1: n}^{*}\right)-T_{n}(\mathrm{Y})\right\} \\
& =\left\{E\left(x_{1: n} x_{1: n}^{*}\right)+E\left(x_{1: n}\right) E\left(\epsilon_{1: n}\right)^{*}+E\left(\epsilon_{1: n}\right) E\left(x_{1: n}\right)^{*}+E\left(\epsilon_{1: n} \epsilon_{1: n}^{*}\right)-T_{n}(\mathrm{Y})\right\} \\
& =\left\{E\left(x_{1: n} x_{1: n}^{*}\right)+E\left(\epsilon_{1: n} \epsilon_{1: n}^{*}\right)-T_{n}(\mathrm{Y})\right\} \sim\left\{E\left(x_{1: n} x_{1: n}^{*}\right)\right\} .
\end{aligned}
$$

Thus, Theorem 4 can be applied in the considered MIMO channel identification problem, that is, it can be used to identify $\Lambda$ and the filter taps $\left\{G_{k}\right\}_{k \in \mathbb{N}}$.

\section{Conclusions}

In ([2], Theorem 4) the (averaged) periodogram method for positive semidefinite Toeplitz matrices was generalized to perturbed block Toeplitz matrices. Moreover, ([2], Theorem 4) was there applied to perturbed positive semidefinite block Toeplitz matrices to solve a statistical signal processing problem: spectral estimation for perturbed WSS vector processes.

In the present paper, ([2], Theorem 4) (Theorem 1) has been applied to perturbed lower triangular block Toeplitz matrices to solve three statistical signal processing problems: parameter estimation for perturbed VAR processes, parameter estimation for perturbed VMA processes, and MIMO channel identification with perturbed additive WSS noise. To solve those problems we have first generalized a result given in [3] on the Cholesky decomposition of Toeplitz matrices to perturbed block Toeplitz matrices.

Author Contributions: Authors are listed in order of their degree of involvement in the work, with the most active contributors listed first. J.G.-G. conceived the research question. All authors were involved in the research and wrote the paper. They have also read and approved the published version of the manuscript.

Funding: This work was supported in part by the Basque Government through the research project "Advanced distributed control for safety and energy efficiency of air transport (CODISAVA)" (KK-2018/00082).

Conflicts of Interest: The authors declare no conflict of interest.

\section{References}

1. Gray, R.M. On the asymptotic eigenvalue distribution of Toeplitz matrices. IEEE Trans. Inf. Theory 1972, IT-18, 725-730. [CrossRef]

2. Gutiérrez-Gutiérrez, J. A modified version of the Pisarenko method to estimate the power spectral density of any asymptotically wide sense stationary vector process. Appl. Math. Comput. 2019, 362, 124526. [CrossRef]

3. Gray, R.M. Toeplitz and circulant matrices: A review. Found. Trends Commun. Inf. Theory 2006, 2, 155-239. [CrossRef]

4. Pisarenko, V.F. On the estimation of spectra by means of non-linear functions of the covariance matrix. Geophys. J. R. Astron. Soc. 1972, 28, 511-531. [CrossRef]

5. Gutiérrez-Gutiérrez, J.; Crespo, P.M. Block Toeplitz matrices: Asymptotic results and applications. Found. Trends Commun. Inf. Theory 2011, 8, 179-257. [CrossRef]

6. Serra, S. Asymptotic results on the spectra of block Toeplitz preconditioned matrices. SIAM J. Matrix Anal. Appl. 1998, 20, 31-44. [CrossRef]

7. Miranda, M.; Tilli, P. Asymptotic spectra of Hermitian block Toeplitz matrices and preconditioning results. SIAM J. Matrix Anal. Appl. 2000, 21, 867-881. [CrossRef]

8. Gutiérrez-Gutiérrez, J.; Crespo, P.M. Asymptotically equivalent sequences of matrices and Hermitian block Toeplitz matrices with continuous symbols: Applications to MIMO systems. IEEE Trans. Inf. Theory 2008, 54, 5671-5680. [CrossRef]

9. Tilli, P. Singular values and eigenvalues of non-Hermitian block Toeplitz matrices. Linear Algebra Appl. 1998, 272, 59-89. [CrossRef]

10. Lancaster, P.; Tismenetsky, M. The Theory of Matrices; Academic Press: Cambridge, MA, USA, 1985.

11. Bernstein, D.S. Matrix Mathematics: Theory, Facts, and Formulas; Princeton University Press: Princeton, NJ, USA, 2009.

12. Gutiérrez-Gutiérrez, J.; Crespo, P.M. Asymptotically equivalent sequences of matrices and multivariate ARMA processes. IEEE Trans. Inf. Theory 2011, 57, 5444-5454. [CrossRef]

13. Gutiérrez-Gutiérrez, J.; Zárraga-Rodríguez, M.; Crespo, P.M.; Insausti, X. Rate distortion function of Gaussian asymptotically WSS vector processes. Entropy 2018, 20, 719. [CrossRef] 
14. Rudin, W. Principles of Mathematical Analysis; McGraw-Hill: New York, NY, USA, 1976.

15. Reinsel, G.C. Elements of Multivariate Time Series Analysis; Springer: Berlin, Germany, 1993.

16. Gazzah, H.; Regalia, P.A.; Delmas, J.P. Asymptotic eigenvalue distribution of block Toeplitz matrices and application to blind SIMO channel identification. IEEE Trans. Inf. Theory 2001, 47, 1243-1251. [CrossRef]

(C) 2020 by the authors. Licensee MDPI, Basel, Switzerland. This article is an open access article distributed under the terms and conditions of the Creative Commons Attribution (CC BY) license (http://creativecommons.org/licenses/by/4.0/). 